\title{
On Certain Properties for Two Classes of Generalized Convex Functions
}

\author{
Mohamed S. S. Ali \\ Department of Mathematics, Faculty of Education, Ain Shams University, Cairo, Egypt \\ Correspondence should be addressed to Mohamed S. S. Ali; mss_ali5@yahoo.com \\ Received 31 March 2016; Accepted 4 May 2016 \\ Academic Editor: Wing-Sum Cheung \\ Copyright (C) 2016 Mohamed S. S. Ali. This is an open access article distributed under the Creative Commons Attribution License, \\ which permits unrestricted use, distribution, and reproduction in any medium, provided the original work is properly cited. \\ Two classes of generalized convex functions in the sense of Beckenbach are considered. For both classes, we show that the existence \\ of support curves implies their generalized convexity and obtain an extremum property of these functions. Furthermore, we \\ establish Hadamard's inequality for them.
}

\section{Introduction}

The convexity of functions plays a central role in many various fields, such as in economics, mechanics, biological system, optimization, and other areas of applied mathematics. Throughout this paper, let $I$ be a nonempty, connected, and bounded subset of $\mathbb{R}$. A real valued function $f(x)$ of a single real variable $x$ defined on $I$ is said to be convex if for all $u, v \in I$ and $t \in[0,1]$ one has the inequality

$$
f(t u+(1-t) v) \leq t f(u)+(1-t) f(v) .
$$

At the beginning of the 20th century, many generalizations of convexity were extensively introduced and investigated in a number of ways by numerous authors in the past and present. One way to generalize the definition of a convex function is to relax the convexity condition (1) (for a comprehensive review, see the monographs [1]).

As it is well known, the notion of the ordinary convexity can be expressed in terms of linear functions. An important direction for generalization of the classical convexity was to replace linear functions by another family of functions. For instance, Beckenbach and Bing [2,3] generalized this situation by replacing the linear functions with a family of continuous functions such that for each pair of points $p_{1}\left(x_{1}, y_{1}\right)$ and $p_{2}\left(x_{2}, y_{2}\right)$ of the plane there exists exactly one member of the family with a graph joining these points.
More precisely, let $\{F(x)\}$ be a family of continuous functions $F(x)$ defined in a real interval $I$. A function $f: I \rightarrow$ $\mathbb{R}$ is said to be sub $F$-function if, for any $u, v \in I$ with $u<v$, there is a unique member of $\{F(x)\}$ satisfying

(i) $F(u)=f(u)$ and $F(v)=f(v)$,

(ii) $f(x) \leq F(x)$ for all $x \in[u, v]$.

The sub $F$-functions possess various properties analogous to those of classical convex functions [2-7]. For example, if $f$ : $I \rightarrow \mathbb{R}$ is sub $F$-function, then, for any $u, v \in I$, the inequality

$$
f(x) \geq F(x)
$$

holds outside the interval $(u, v)$.

Theorem 1. A sub F-function $f: I \rightarrow \mathbb{R}$ has finite left and right derivatives $f_{-}^{\prime}(x), f_{+}^{\prime}(x)$ at every point $x \in I$, and $f_{-}^{\prime}(x) \leq$ $f_{+}^{\prime}(x)$ for all $x \in I$.

Property 1. Under the assumptions of Theorem 1, the function $f$ is continuously differentiable on $I$ with the exception of an at-most countable set.

Of course mathematicians were able before 1937 to generalize the notion of convex functions [8-10]. Full details could be found in two classic books $[11,12]$ or in the new monographs like [13].

In this paper, we deal just with generalized convexity in the sense of Beckenbach. For particular choices of the 
two-parameter family $\{F(x)\}$, one considers two classes of generalized convex functions:

(i) $F(x)=H(x)=A \cosh p x+B \sinh p x$, where $p$ is a fixed constant.

(ii) $F(x)=E(x)=A e^{B x}$.

The following double inequality

$$
f\left(\frac{a+b}{2}\right) \leq \frac{1}{b-a} \int_{a}^{b} f(x) d x \leq \frac{f(a)+f(b)}{2}
$$

is well known in the literature as Hadamard's inequality or, as it is quoted for historical reasons [14], the Hermite-Hadamard inequality, where $f: I \rightarrow \mathbb{R}$ is a convex function and $a, b \in I$ with $a<b$. This inequality has evoked the interest of many mathematicians; for new generalizations, extensions, and numerous applications, see, for example, [15-18].

A basic theorem [11] in the theory of convex functions states that a necessary and sufficient condition in order that the function $f: I \rightarrow \mathbb{R}$ be convex is that there is at least one line of support for $f$ at each point $x$ in $I$.

In this paper, we prove analogues of this result for the classes of sub $H$-functions and $E$-functions. We also extend the extremum property (as stated in [19]) and the HermiteHadamard inequality.

\section{Definitions and Preliminary Results}

Inspired by these investigations, let us now introduce the basic definitions and results for the preceding two classes, respectively, of generalized convex functions in the sense of Beckenbach as will be used later in this note.

Definition 2. A function $f: I \rightarrow \mathbb{R}$ is said to be sub $H$ function on $I$, if for any arbitrary closed subinterval $[u, v]$ of $I$ the graph of $f(x)$ for $x \in[u, v]$ lies nowhere above the function

$$
H(x)=A \cosh p x+B \sinh p x,
$$

where $A$ and $B$ are chosen such that $H(u)=f(u)$ and $H(v)=$ $f(v)$.

Equivalently, for all $x \in[u, v]$

$$
\begin{aligned}
f(x) & \leq H(x) \\
& =\frac{f(u) \sinh p(v-x)+f(v) \sinh p(x-u)}{\sinh p(v-u)} .
\end{aligned}
$$

Note that the condition $f^{\prime \prime}(x)-p^{2} f(x) \geq 0$ for all $x$ in $I$ is necessary and sufficient in order that the twice differentiable function $f: I \rightarrow \mathbb{R}$ be sub $H$-function on $I$.

Definition 3. Let $f: I \rightarrow \mathbb{R}$ be a sub $H$-function.

A function

$$
S_{u}(x)=A \cosh p x+B \sinh p x
$$

is said to be a supporting function for $f(x)$ at the point $u \in I$, if

$$
\begin{aligned}
& S_{u}(u)=f(u), \\
& S_{u}(x) \leq f(x) \quad \forall x \in I .
\end{aligned}
$$

That is, if $f(x)$ and $S_{u}(x)$ agree at $x=u$, the graph of $f(x)$ does not lie under the support curve.

Proposition 4. If $f: I \rightarrow \mathbb{R}$ is a differentiable sub H-function, then the supporting function for $f(x)$ at the point $u \in I$ has the form

$$
S_{u}(x)=f(u) \cosh p(x-u)+f^{\prime}(u) \sinh p(x-u) .
$$

Proof. The supporting function $S_{u}(x)$ for $f(x)$ at the point $u \in$ I can be described as follows:

$$
S_{u}(x)=\lim _{v \rightarrow u} H(x),
$$

where $v \in I$ and

$$
f(x) \geq H(x), \quad \forall x \in I \backslash(u, v) .
$$

Then, taking the limit of both sides as $v \rightarrow u$ and from (5), one obtains

$$
\begin{aligned}
f(x) & \geq S_{u}(x)=\lim _{v \rightarrow u} H(x) \\
& =\lim _{v \rightarrow u} \frac{f(u) \sinh p(v-x)+f(v) \sinh p(x-u)}{\sinh p(v-u)} \\
& =f(u) \cosh p(x-u)+f^{\prime}(u) \sinh p(x-u) .
\end{aligned}
$$

Thus, the claim follows.

Definition 5. A positive function $f: I \rightarrow(0, \infty)$ is called sub $E$-function on $I$, if for any $u, v \in I$ with $u<v$ the graph of $f(x)$ for $u \leq x \leq v$ lies on or under the function

$$
E(x)=A e^{B x},
$$

where $A$ and $B$ are taken so that $E(u)=f(u)$, and $E(v)=$ $f(v)$.

Equivalently, for all $x \in[u, v]$

$$
\begin{aligned}
f(x) & \leq E(x) \\
& =\exp \left[\frac{(v-x) \ln f(u)+(x-u) \ln f(v)}{v-u}\right] .
\end{aligned}
$$

Note the following:

(1) There is more than one formula for the function $E(x)$ other than that stated in (13); for example,

$$
E(x)=f(u) e^{B(x-u)} ; \quad B=\frac{\ln f(v)-\ln f(u)}{v-u},
$$

or in a multiplicative form

$$
E(x)=[f(u)]^{((v-x) /(v-u))} \cdot[f(v)]^{((x-u) /(v-u))} .
$$

(2) Let $f: I \rightarrow(0, \infty)$ be a two-time continuously differentiable function. Then, $f$ is sub $E$-function on $I$ if and only if $f(x) f^{\prime \prime}(x)-\left(f^{\prime}(x)\right)^{2} \geq 0$ for all $x \in I$. 
Definition 6. Let $f: I \rightarrow(0, \infty)$ be a sub $E$-function. A function

$$
T_{u}(x)=A e^{B x}
$$

is said to be a supporting function for $f(x)$ at the point $u \in I$, if

$$
\begin{aligned}
& T_{u}(u)=f(u), \\
& T_{u}(x) \leq f(x) \quad \forall x \in I .
\end{aligned}
$$

That is, if $f(x)$ and $T_{u}(x)$ agree at $x=u$, the graph of $f(x)$ lies on or above the support curve.

Proposition 7. If $f: I \rightarrow(0, \infty)$ is a differentiable sub Efunction, then the supporting function for $f(x)$ at the point $u \in$ I has the form

$$
T_{u}(x)=f(u) \exp \left[(x-u) \frac{f^{\prime}(u)}{f(u)}\right] .
$$

Proof. The supporting function $T_{u}(x)$ for $f(x)$ at the point $u \in I$ can be described as follows:

$$
T_{u}(x)=\lim _{v \rightarrow u} E(x)
$$

where $v \in I$ and

$$
f(x) \geq E(x), \quad \forall x \in I \backslash(u, v) .
$$

Then, taking the limit of both sides as $v \rightarrow u$ and from (14), one obtains

$$
\begin{aligned}
f(x) & \geq T_{u}(x)=\lim _{v \rightarrow u} E(x)=\lim _{v \rightarrow u}\left[f(u) e^{B(x-u)}\right] \\
& =f(u) \exp \left[(x-u) \frac{f^{\prime}(u)}{f(u)}\right] .
\end{aligned}
$$

Thus, the claim follows.

In the literature, the logarithmic mean of the positive real numbers $p, q$ is defined as

$$
L(p, q)= \begin{cases}\frac{q-p}{\ln q-\ln p} & \text { if } p \neq q \\ p & \text { if } p=q .\end{cases}
$$

The logarithmic mean proves useful in engineering problems involving heat and mass transfer.

\section{Results}

Theorem 8. A function $f: I \rightarrow \mathbb{R}$ is sub H-function on $I$ if and only if there exists a supporting function for $f(x)$ at each point $x$ in $I$.

Proof. The necessity is an immediate consequence of Bonsall [20].

To prove the sufficiency, let $x$ be an arbitrary point in $I$ and $f$ has a supporting function at this point. For convenience, we will write the supporting function in the following form:

$$
S_{x}(z)=f(x) \cosh p(z-x)+K_{x, f} \sinh p(z-x),
$$

where $K_{x, f}$ is a fixed real number depending on $x$ and $f$.

From Definition 3, one has

$$
\begin{aligned}
& S_{x}(x)=f(x), \\
& S_{x}(z) \leq f(z) \quad \forall z \in I .
\end{aligned}
$$

It follows that

$$
\begin{aligned}
f(x) \cosh p(z-x)+K_{x, f} \sinh p(z-x) \leq f(z) & \\
& \forall z \in I .
\end{aligned}
$$

For all $u, v \in I$ with $u<v$ and $\lambda, \mu \geq 0$ with $\lambda+\mu=1$ let

$$
x=\lambda u+\mu v .
$$

Applying (25) twice at $z=u$ and at $z=v$ yields

$$
\begin{aligned}
& f(x) \cosh p(u-x)+K_{x, f} \sinh p(u-x) \leq f(u), \\
& f(x) \cosh p(v-x)+K_{x, f} \sinh p(v-x) \leq f(v) .
\end{aligned}
$$

Multiplying the first inequality by $\sinh p \lambda(v-u)$ and the second by $\sinh p \mu(v-u)$ and adding them, we obtain

$$
\begin{aligned}
& f(x)[\sinh p \lambda(v-u) \cosh p(u-x) \\
& -\cosh p(v-x) \sinh p \mu(u-v)] \\
& +K_{x, f}[\sinh p \lambda(v-u) \sinh p(u-x) \\
& -\sinh p(v-x) \sinh p \mu(u-v)] \leq f(u) \\
& \cdot \sinh p \lambda(v-u)+f(v) \sinh p \mu(v-u) .
\end{aligned}
$$

Consequently,

$$
\begin{array}{r}
f(x) \leq \frac{f(u) \sinh p(v-x)+f(v) \sinh p(x-u)}{\sin \rho(v-u)} \\
\forall x \in[u, v],
\end{array}
$$

which proves that the function $f(x)$ is sub $H$-function on $I$. Hence, the theorem follows.

Remark 9. For a sub $H$-function $f: I \rightarrow \mathbb{R}$, the constant $K_{x, f}$ in the foregoing theorem is equal to $f^{\prime}(x)$ if $f$ is differentiable at the point $x \in I$; otherwise, $K_{x, f} \in\left[f_{-}^{\prime}(x), f_{+}^{\prime}(x)\right]$.

Theorem 10. Let $f: I \rightarrow \mathbb{R}$ be a sub $H$-function and $a, b \in I$ with $a<b$, and let $S_{u}(x)$ be a supporting function for $f(x)$ at the point $u \in[a, b]$. Then, the function

$$
G(u)=\int_{a}^{b}\left[f(x)-S_{u}(x)\right] d x
$$

has a minimum value at $u=(a+b) / 2$. 
Proof. From Definition 3, we have

$$
\begin{aligned}
& S_{u}(u)=f(u), \\
& S_{u}(x) \leq f(x) \quad \forall x \in[a, b],
\end{aligned}
$$

and $S_{u}(x)$ can be written in the form

$$
S_{u}(x)=f(u) \cosh p(x-u)+K_{u, f} \sinh p(x-u) .
$$

Using (33), one obtains

$$
\begin{aligned}
& \int_{a}^{b} S_{u}(x) d x=f(u) \int_{a}^{b} \cosh p(x-u) d x \\
& +K_{u, f} \int_{a}^{b} \sinh p(x-u) d x=\frac{2}{p} \\
& \cdot \sinh p\left(\frac{b-a}{2}\right)\left\{f(u) \cosh p\left[\left(\frac{a+b}{2}\right)-u\right]\right. \\
& \left.+K_{u, f} \sinh p\left[\left(\frac{a+b}{2}\right)-u\right]\right\}=\frac{2}{p} \\
& \cdot \sinh p\left(\frac{b-a}{2}\right) S_{u}\left(\frac{a+b}{2}\right) .
\end{aligned}
$$

Consequently,

$$
\begin{aligned}
G(u)= & \int_{a}^{b} f(x) d x \\
& -\frac{2}{p} \sinh p\left(\frac{b-a}{2}\right) S_{u}\left(\frac{a+b}{2}\right) .
\end{aligned}
$$

Using (31) at $u=(a+b) / 2$, the function $G(u)$ becomes

$$
\begin{aligned}
G\left(\frac{a+b}{2}\right)= & \int_{a}^{b} f(x) d x \\
& -\frac{2}{p} \sinh p\left(\frac{b-a}{2}\right) f\left(\frac{a+b}{2}\right) .
\end{aligned}
$$

But from (32), we observe

$$
S_{u}\left(\frac{a+b}{2}\right) \leq f\left(\frac{a+b}{2}\right) \quad \forall u \in(a, b) .
$$

Now using (35) and (36), it follows that

$$
G(u) \geq G\left(\frac{a+b}{2}\right) \quad \forall u \in(a, b) .
$$

Hence, the minimum value of the function $G(u)$ occurs at $u=$ $(a+b) / 2$.

Theorem 11. Suppose $f: I \rightarrow \mathbb{R}$ is sub $H$-function, and $a, b \in$ I with $a<b$.

Then, one has the inequality

$$
\begin{gathered}
\frac{2}{p} f\left(\frac{a+b}{2}\right) \sinh p\left(\frac{b-a}{2}\right) \leq \int_{a}^{b} f(x) d x \\
\quad \leq \frac{1}{p}[f(a)+f(b)] \tanh p\left(\frac{b-a}{2}\right) .
\end{gathered}
$$

Proof. Let $u$ be an arbitrary point in $(a, b)$. As $f(x)$ is a sub $H$-function, then from Definitions 2 and 3 we observe that the graph of $f(x)$ lies nowhere above the function

$$
H(x)=\frac{f(a) \sinh p(b-x)+f(b) \sinh p(x-a)}{\sinh p(b-a)}
$$

and nowhere below any supporting function:

$$
S_{u}(x)=f(u) \cosh p(x-u)+K_{u, f} \sinh p(x-u),
$$

at the point $u \in(a, b)$.

Hence,

$$
S_{u}(x) \leq f(x) \leq H(x) \quad x \in[a, b],
$$

and thus

$$
\int_{a}^{b} S_{u}(x) d x \leq \int_{a}^{b} f(x) d x \leq \int_{a}^{b} H(x) d x .
$$

Using (40), one has

$$
\begin{aligned}
& \int_{a}^{b} H(x) d x \\
& \quad=\frac{1}{\sinh p(b-a)}\left[f(a) \int_{a}^{b} \sinh p(b-x) d x\right. \\
& \left.+f(b) \int_{a}^{b} \sinh p(x-a) d x\right]=\frac{1}{p}[f(a)+f(b)] \\
& \cdot\left[\frac{\cosh p(b-a)-1}{\sinh p(b-a)}\right]=\frac{1}{p}[f(a)+f(b)] \\
& \quad \cdot \tanh p\left(\frac{b-a}{2}\right) .
\end{aligned}
$$

Using (41) and (34), one obtains

$$
\int_{a}^{b} S_{u}(x) d x=\frac{2}{p} S_{u}\left(\frac{a+b}{2}\right) \sinh p\left(\frac{b-a}{2}\right) .
$$

But from (32), we observe that

$$
S_{u}\left(\frac{a+b}{2}\right) \leq f\left(\frac{a+b}{2}\right) \quad \forall u \in(a, b) .
$$

Taking the maximum of the term $\int_{a}^{b} S_{u}(x) d x \leq$ $\int_{a}^{b} f(x) d x$ in (43) and (45) for $u \in(a, b)$ and from (46), it follows that

$$
\begin{aligned}
\int_{a}^{b} f(x) d x & \geq \max _{a<u<b}\left\{\int_{a}^{b} S_{u}(x) d x\right\} \\
& =\frac{2}{p} \max _{a<u<b}\left\{S_{u}\left(\frac{a+b}{2}\right)\right\} \sinh p\left(\frac{b-a}{2}\right) \\
& =\frac{2}{p} f\left(\frac{a+b}{2}\right) \sinh p\left(\frac{b-a}{2}\right) .
\end{aligned}
$$

Hence, from (43), (44), and (47), we get the desired inequality (39). 
Theorem 12. A function $f: I \rightarrow(0, \infty)$ is a sub E-function on $I$ if and only if there exists a supporting function for $f(x)$ at each point $x$ in $I$.

Proof. The necessity is an immediate consequence of Bonsall [20].

To prove the sufficiency, let $x$ be an arbitrary point in $I$ and $f$ has a supporting function at this point. For convenience, we will write the supporting function in the following form:

$$
T_{x}(z)=f(x) \exp \left[(z-x) \frac{M_{x, f}}{f(x)}\right],
$$

where $M_{x, f}$ is a fixed real number depending on $x$ and $f$.

From Definition 6, one has

$$
\begin{aligned}
& T_{x}(x)=f(x), \\
& T_{x}(z) \leq f(z) \quad \forall z \in I .
\end{aligned}
$$

It follows that

$$
f(x) \exp \left[(z-x) \frac{M_{x, f}}{f(x)}\right] \leq f(z) \quad \forall z \in I .
$$

As $f(x)$ is a positive function, we infer that

$$
(z-x) \frac{M_{x, f}}{f(x)} \leq \ln f(z)-\ln f(x) \quad \forall z \in I .
$$

For all $u, v \in I$ with $u<v$ and $\lambda, \mu \geq 0$ with $\lambda+\mu=1$ let

$$
x=\lambda u+\mu v .
$$

Applying (51) twice at $z=u$ and at $z=v$ yields

$$
\begin{aligned}
& (u-x) \frac{M_{x, f}}{f(x)} \leq \ln f(u)-\ln f(x), \\
& (v-x) \frac{M_{x, f}}{f(x)} \leq \ln f(v)-\ln f(x) .
\end{aligned}
$$

Multiplying the first inequality by $\lambda(v-u)$ and the second by $\mu(v-u)$ and adding them, we obtain

$$
\begin{gathered}
\frac{M_{x, f}}{f(x)}[\lambda(v-u)(u-x)+\mu(v-u)(v-x)] \\
\leq \lambda(v-u)[\ln f(u)-\ln f(x)] \\
\quad+\mu(v-u)[\ln f(v)-\ln f(x)] .
\end{gathered}
$$

Consequently,

$$
\begin{array}{r}
f(x) \leq \exp \left[\frac{(v-x) \ln f(u)+(x-u) \ln f(v)}{v-u}\right] \\
\forall x \in[u, v],
\end{array}
$$

which proves that the function $f(x)$ is a sub $E$-function on $I$.

Hence, the theorem follows.
Remark 13. For a sub E-function $f: I \rightarrow(0, \infty)$, the constant $M_{x, f}$ in the preceding theorem is equal to $f^{\prime}(x)$ if $f$ is differentiable at the point $x \in I$; otherwise, $f_{-}^{\prime}(x) \leq M_{x, f} \leq$ $f_{+}^{\prime}(x)$.

Theorem 14. Let $f: I \rightarrow(0, \infty)$ be a sub E-function, and $a, b \in I$ with $a<b$ and let $T_{u}(x)$ be a supporting function for $f(x)$ at the point $u \in[a, b]$. Then, the function

$$
G(u)=\int_{a}^{b}\left[f(x)-T_{u}(x)\right] d x
$$

has a minimum value at $u=(a+b) / 2$.

Proof. From Definition 6, we have

$$
\begin{aligned}
& T_{u}(u)=f(u), \\
& T_{u}(x) \leq f(x) \quad \forall x \in[a, b],
\end{aligned}
$$

and $T_{u}(x)$ can be written in the form

$$
T_{u}(x)=f(u) \exp \left[(x-u) \frac{M_{u, f}}{f(u)}\right] .
$$

Using (59), one obtains

$$
\begin{gathered}
\int_{a}^{b} T_{u}(x) d x=f(u) \int_{a}^{b} \exp \left[(x-u) \frac{M_{u, f}}{f(u)}\right] d x \\
=\frac{f(u)}{M_{u, f}}\left[f(u) \exp \left[\left(\frac{a+b}{2}-u\right) \frac{M_{u, f}}{f(u)}\right]\right] \\
\cdot\left[\exp \left[\left(\frac{b-a}{2}\right) \frac{M_{u, f}}{f(u)}\right]\right. \\
\left.-\exp \left[\left(\frac{a-b}{2}\right) \frac{M_{u, f}}{f(u)}\right]\right]=\frac{2 f(u)}{M_{u, f}} \\
\cdot \sinh \left[\left(\frac{b-a}{2}\right) \frac{M_{u, f}}{f(u)}\right] T_{u}\left(\frac{a+b}{2}\right) .
\end{gathered}
$$

Consequently,

$$
\begin{aligned}
G(u)=\int_{a}^{b} f(x) d x & -M T_{u}\left(\frac{a+b}{2}\right) ; \\
M & =\frac{2 f(u)}{M_{u, f}} \sinh \left[\left(\frac{b-a}{2}\right) \frac{M_{u, f}}{f(u)}\right] .
\end{aligned}
$$

One can easily check that $M$ does not depend on $u$. That is, $M$ depends only on $a$ and $b$.

Using (57) at $u=(a+b) / 2$, the function $G(u)$ becomes

$$
G\left(\frac{a+b}{2}\right)=\int_{a}^{b} f(x) d x-M f\left(\frac{a+b}{2}\right) .
$$

But from (58), we observe

$$
T_{u}\left(\frac{a+b}{2}\right) \leq f\left(\frac{a+b}{2}\right) \quad \forall u \in(a, b) .
$$


Now using (61) and (62), it follows that

$$
G(u) \geq G\left(\frac{a+b}{2}\right) \quad \forall u \in(a, b) .
$$

Hence, the minimum value of the function $G(u)$ occurs at $u=(a+b) / 2$.

Theorem 15. Assume $f: I \rightarrow(0, \infty)$ is a sub E-function, and $a, b \in I$ with $a<b$.

Then, one has the inequality

$$
f\left(\frac{a+b}{2}\right) \leq \frac{1}{b-a} \int_{a}^{b} f(x) d x \leq L(f(a), f(b)) .
$$

Proof. Let $u$ be an arbitrary point in $(a, b)$. As $f(x)$ is a sub $E$ function, then from Definitions 5 and 6 we observe that the graph of $f(x)$ lies nowhere above the function

$$
E(x)=f(a) e^{B(x-a)} ; \quad B=\frac{\ln f(b)-\ln f(a)}{b-a}
$$

and nowhere below any supporting function:

$$
T_{u}(x)=f(u) \exp \left[(x-u) \frac{M_{u, f}}{f(u)}\right],
$$

at the point $u \in(a, b)$.

Thus,

$$
\begin{aligned}
T_{u}(x) & \leq f(x) \leq E(x) \quad x \in[a, b], \\
\frac{1}{b-a} \int_{a}^{b} T_{u}(x) d x & \leq \frac{1}{b-a} \int_{a}^{b} f(x) d x \\
& \leq \frac{1}{b-a} \int_{a}^{b} E(x) d x .
\end{aligned}
$$

Using (66), one has

$$
\begin{aligned}
\frac{1}{b-a} \int_{a}^{b} E(x) d x & =\frac{f(a)}{b-a} \int_{a}^{b} e^{B(x-a)} d x \\
& =\frac{f(a)}{\ln f(b)-\ln f(a)}\left[\frac{f(b)}{f(a)}-1\right] \\
& =L(f(a), f(b)) .
\end{aligned}
$$

Using (67) and (60), one obtains

$$
\begin{gathered}
\frac{1}{b-a} \int_{a}^{b} T_{u}(x) d x=\left(\frac{2}{b-a}\right) \frac{f(u)}{M_{u, f}} \\
\cdot \sinh \left[\left(\frac{b-a}{2}\right) \frac{M_{u, f}}{f(u)}\right] T_{u}\left(\frac{a+b}{2}\right) .
\end{gathered}
$$

It is easy to notice that

$$
\frac{1}{b-a} \int_{a}^{b} T_{u}(x) d x \geq T_{u}\left(\frac{a+b}{2}\right) .
$$

But from (58), we observe that

$$
T_{u}\left(\frac{a+b}{2}\right) \leq f\left(\frac{a+b}{2}\right) \quad \forall u \in(a, b) .
$$

Taking the maximum of the term $(1 /(b-a)) \int_{a}^{b} T_{u}(x) d x \leq$ $(1 /(b-a)) \int_{a}^{b} f(x) d x$ in (68) and (71) for $u \in(a, b)$ and from (72), it follows that

$$
\begin{aligned}
\frac{1}{b-a} \int_{a}^{b} f(x) d x & \geq \max _{a<u<b}\left\{\frac{1}{b-a} \int_{a}^{b} T_{u}(x) d x\right\} \\
& \geq \max _{a<u<b}\left\{T_{u}\left(\frac{a+b}{2}\right)\right\} \\
& =f\left(\frac{a+b}{2}\right) .
\end{aligned}
$$

Hence, from (68), (69), and (73), we get the required inequality (65).

Remark 16. Recall that a positive function $f: I \rightarrow(0, \infty)$ is said to be log-convex or multiplicatively convex if $\log f(x)$ is convex, equivalently, if for all $u, v \in I$ and $t \in[0,1]$ one has the inequality

$$
f(t u+(1-t) v) \leq[f(u)]^{t} \cdot[f(v)]^{1-t}
$$

using the following substitution:

$$
t=\frac{v-x}{v-u} .
$$

Then, from (15), the function $E(x)$ becomes

$$
E(x)=E(t u+(1-t) v)=[f(u)]^{t} \cdot[f(v)]^{1-t} .
$$

Thus, from Definition 5, it follows that a function $f: I \rightarrow$ $(0, \infty)$ is a sub $E$-function on $I$, if for all $u, v \in I$ and $t \in[0,1]$ one has the inequality

$$
\begin{aligned}
f(t u+(1-t) v) & =f(x) \leq E(x) \\
& =[f(u)]^{t} \cdot[f(v)]^{1-t} .
\end{aligned}
$$

Therefore, from (74) and (77), we conclude that the sub $E$-functions are exactly the "standard" log-convex functions. On the other hand, this class of sub $E$-functions can be considered as another point of view for log-convex functions.

\section{Conclusion}

Various generalizations of convex functions have appeared in the literature. In this paper, two classes of generalized convex functions in the sense of Beckenbach are considered. Some properties and inequalities for these classes are established.

\section{Competing Interests}

The author declares that there is no conflict of interests regarding the publication of this paper. 


\section{References}

[1] I. V. Konnov, D. T. Luc, and A. M. Rubinov, Generalized Convexity and Related Topics, Springer, Berlin, Germany, 2007.

[2] E. F. Beckenbach, "Generalized convex functions," Bulletin of the American Mathematical Society, vol. 43, no. 6, pp. 363-371, 1937.

[3] E. F. Beckenbach and R. H. Bing, "On generalized convex functions," Transactions of the American Mathematical Society, vol. 58 , pp. 220-230, 1945.

[4] E. F. Bechenbach, "Convex functions," Bulletin of the American Mathematical Society, vol. 54, pp. 439-460, 1948.

[5] J. W. Green, "Support, convergence, and differentiability properties of generalized convex functions," Proceedings of the American Mathematical Society, vol. 4, pp. 391-396, 1953.

[6] M. M. Peixoto, "On the existence of derivatives of generalized convex functions," Summa Brasilian Mathematics, vol. 2, no. 3, pp. 35-42, 1948.

[7] M. M. Peixoto, "Generalized convex functions and second order differential inequalities," Bulletin of the American Mathematical Society, vol. 55, pp. 563-572, 1949.

[8] E. Phragmén and E. Lindelöf, "Sur une extension d'un principe classique de l'analyse et sur quelques propriétés des fonctions monogènes dans le voisinage d'un point singulier," Acta Mathematica, vol. 31, no. 1, pp. 381-406, 1908.

[9] G. Pólya, "Untersuchungen über Lücken und singularitäten von potenzreihen," Mathematische Zeitschrift, vol. 29, no. 1, pp. 549640, 1929.

[10] G. Valiron, "Fonctions convexes et fonctions entières," Bulletin de la Société Mathématique de France, vol. 60, pp. 178-287, 1932.

[11] A. W. Roberts and D. E. Varberg, Convex Functions, Academic Press, London, UK, 1973.

[12] R. T. Rockafellar, Convex Analysis, Princeton University Press, Princeton, NJ, USA, 1970.

[13] C. P. Niculescu and L. E. Persson, Convex Functions and their Applications. A Contemporary Approach, Springer, New York, NY, USA, 2006.

[14] D. S. Mitrinović and I. B. Lacković, "Hermite and convexity," Aequationes Mathematicae, vol. 28, no. 3, pp. 229-232, 1985.

[15] İ. İscan and M. Kunt, "Hermite-Hadamard-Fejer type inequalities for harmonically convex functions via fractional integrals," RGMIA Research Report Collection, vol. 18, pp. 1-16, 2015.

[16] E. Set, M. E. Özdemir, and S. S. Dragomir, "On the hermitehadamard inequality and other integral inequalities involving two functions," Journal of Inequalities and Applications, vol. 2010, Article ID 148102, 9 pages, 2010.

[17] Y. Shuang, H.-P. Yin, and F. Qi, "Hermite-Hadamard type integral inequalities for geometric-arithmetically s-convex functions," Analysis, vol. 33, no. 2, pp. 197-208, 2013.

[18] X.-M. Zhang, Y.-M. Chu, and X.-H. Zhang, "The HermiteHadamard type inequality of GA-convex functions and its applications," Journal of Inequalities and Applications, vol. 2010, Article ID 507560, 11 pages, 2010.

[19] M. J. Miles, "An extremum property of convex functions," The American Mathematical Monthly, vol. 76, pp. 921-922, 1969.

[20] F. F. Bonsall, "The characterization of generalized convex functions," The Quarterly Journal of Mathematics. Oxford Series, vol. 1, no. 1, pp. 100-111, 1950. 


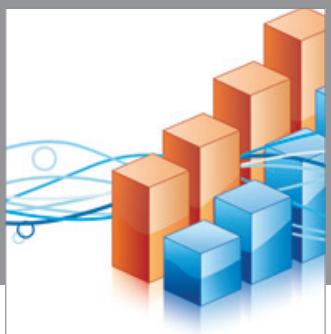

Advances in

Operations Research

vatem alat4

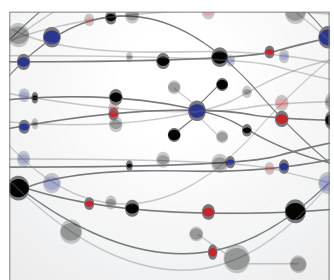

\section{The Scientific} World Journal
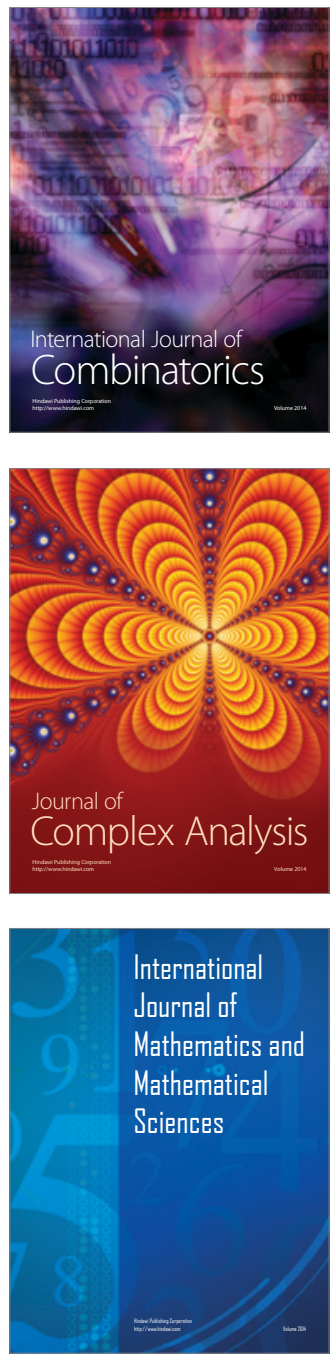
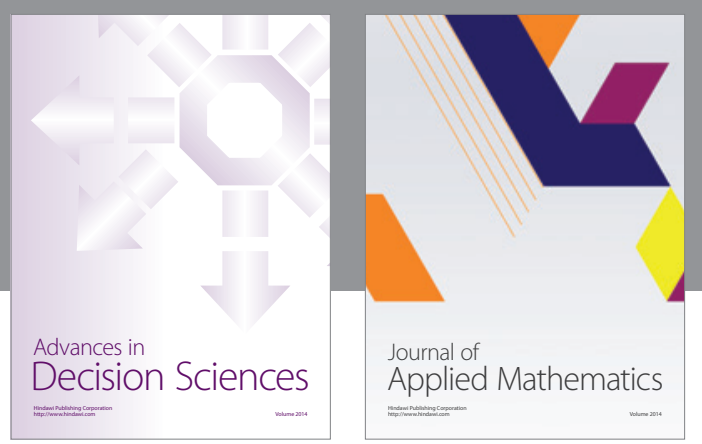

Algebra

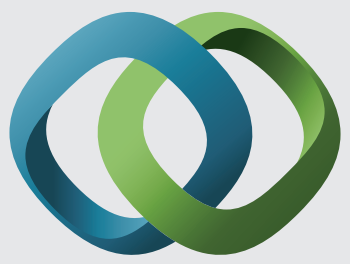

\section{Hindawi}

Submit your manuscripts at

http://www.hindawi.com
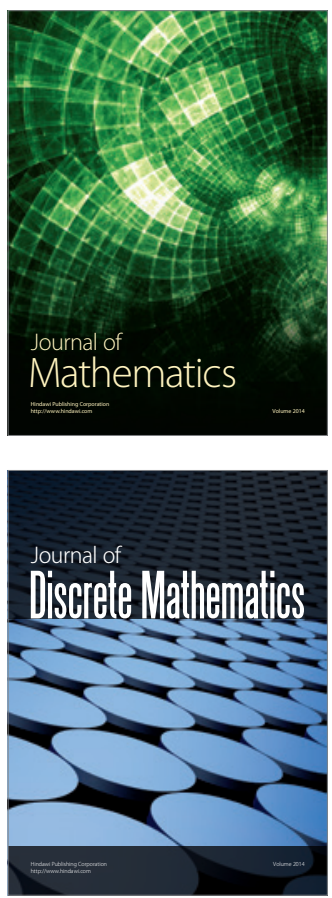

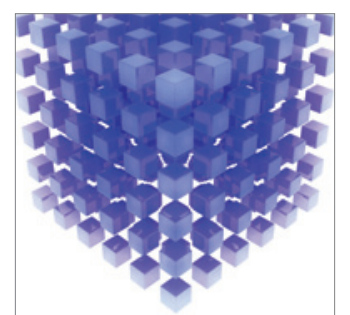

Mathematical Problems in Engineering
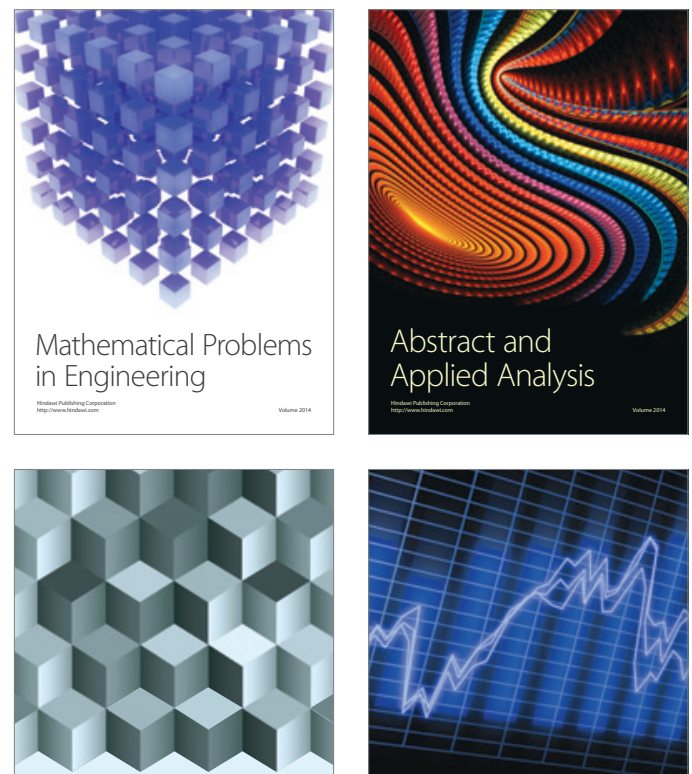

Journal of

Function Spaces

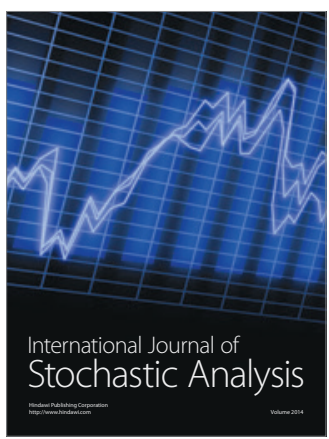

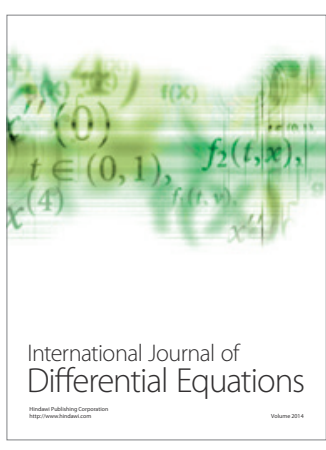
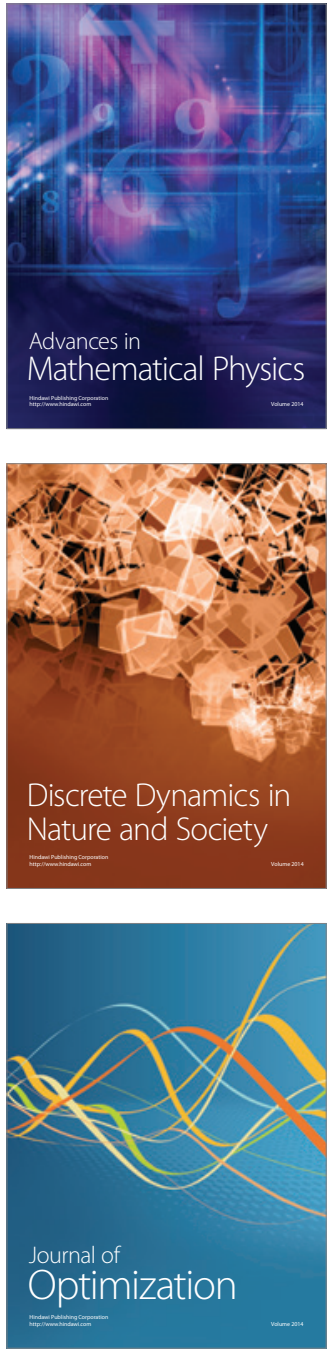